\begin{tabular}{|c|c|}
\hline Title & Electroabsorption spectra of PbSe nanocry stal quantum dots \\
\hline Author(s) & Liu, Xiaoming; limori, Toshifumi; Ohshima, Ruriko; Nakabay ashi, Takakazu; Ohta, Nobuhiro \\
\hline Citation & $\begin{array}{l}\text { A pplied Physics Letters, 98(16), } 161911 \\
\text { https://doi.org/10.1063/1.3583450 }\end{array}$ \\
\hline Issue Date & 2011-04-18 \\
\hline Doc URL & http:/hdl.handle.net/2115/45542 \\
\hline Rights & $\begin{array}{l}\text { Copyright } 2011 \text { A merican Institute of Phy sics. This article may be downloaded for personal use only. A ny other use } \\
\text { requires prior permission of the author and the A merican Institute of Physics. The following article appeared in A ppl. } \\
\text { Phys. Lett. 98, 161911 (2011) and may be found at https://dx.doi.org/10.1063/1.3583450 }\end{array}$ \\
\hline Type & article \\
\hline File Information & APL98-16_161911.pdf \\
\hline
\end{tabular}

Instructions for use 


\title{
Electroabsorption spectra of PbSe nanocrystal quantum dots
}

\author{
Xiaoming Liu, ${ }^{1,2}$ Toshifumi limori, ${ }^{1}$ Ruriko Ohshima, ${ }^{1}$ Takakazu Nakabayashi, ${ }^{1}$ and \\ Nobuhiro Ohta ${ }^{1, a)}$ \\ ${ }^{1}$ Research Institute for Electronic Science, Hokkaido University, Sapporo 001-0020, Japan \\ ${ }^{2}$ School of Environment and Chemical Engineering, Nanchang Hangkong University, Nanchang 330063, \\ People's Republic of China
}

(Received 11 November 2010; accepted 3 April 2011; published online 22 April 2011)

\begin{abstract}
The quantum-confined Stark effect of PbSe nanocrystal quantum dots (QDs) in a polymer film has been studied for the near-infrared absorption in the region of 1300-1600 nm (corresponding to diameters of 4.0-7.0 nm) by using electric field modulation spectroscopy. Electroabsorption spectra of QDs show the electric-field-induced energy shift in the exciton state, resulting in the spectral broadening given by the second derivative of the absorption spectra. It is shown that the magnitude of the change in electric dipole moment following excitation into the first exciton state increases with an increase in the size of QDs. (C) 2011 American Institute of Physics. [doi:10.1063/1.3583450]
\end{abstract}

Quantum confinement transforms the band structure of the bulk semiconductor into a series of discrete transitions. As a result, a number of physical phenomena observed in atomic physics have also been seen in quantum dots (QDs), including quantum-confined Stark effect (QCSE), which can be applied to ultrafast optoelectronics, such as optical switches, electro-optical modulators, or detectors. ${ }^{1-3}$ The QCSE was reported in the visible spectral range for single QDs of CdSe, ZnSe, CdS, $\mathrm{CdS}_{\mathrm{x}} \mathrm{Se}_{1-\mathrm{x}}$, InGaN, etc. ${ }^{1-6}$ However, there have been very few studies on QCSE of the QDs which show the near-infrared (NIR) absorption, although it is critical to data communication demand sources, detectors, self-electro-optic effect devices, and modulators in the extended telecommunications wavelength band. ${ }^{7,8}$

Recently, lead selenide (PbSe), i.e., a direct band gap semiconductor material with a rather small bulk band gap $(0.27 \mathrm{eV})$ and a larger excitation Bohr radius $(46 \mathrm{~nm})$, has received much attention owing to its great potential applications in NIR lasers, solar cells, biomedical imaging and labeling, optical switches, telecommunications, photodetectors, modulators, etc. ${ }^{9-11}$ However, electroabsorption (E-A) spectra of $\mathrm{PbSe}$ QDs have not been reported yet. In this letter, E-A spectra of PbSe QDs dispersed in a poly(methyl methacrylate) (PMMA) film which show NIR absorption in the region of 1300-1600 nm (corresponding to diameters of $4.0-7.0 \mathrm{~nm})$ are reported.

$\mathrm{PbSe}$ nanocrystals (NCs) were synthesized by a noncoordinating solvent method. ${ }^{12} \mathrm{PbSe}$ NCs with different diameters were obtained by varying the reaction time. After purification, a certain amount of chloroform solution of a mixture of PbSe NCs and PMMA was cast on an indium-tin oxide (ITO) coated quartz substrate by a spin coating method. Then, a semitransparent aluminum (Al) film was deposited on the dried polymer film by a vacuum vapor deposition technique. The ITO and Al films were used as electrodes. The X-ray diffraction (XRD) measurements were carried out on a Rigaku-Dmax 2500 diffractometer using $\mathrm{Cu} K \alpha$ radiation $(\lambda=0.15405 \mathrm{~nm})$. The size of the samples was estimated using a scanning transmission electron micro-

\footnotetext{
${ }^{\text {a) }}$ Author to whom correspondence should be addressed. Electronic mail: nohta@es.hokudai.ac.jp.
}

scope (Hitachi HD-2000 STEM). The instruments used for the measurements of the absorption and E-A spectra were the same as those described previously. ${ }^{13}$ All the measurements were done at room temperature.

Figure 1 shows the representative XRD pattern for $\mathrm{PbSe}$ NCs sample with exciton absorption band at $1450 \mathrm{~nm}$. All the diffraction peaks can be basically indexed to the standard data of PbSe [Joint Committee on Powder Diffraction Standards (JCPDS 65-2491)]. The broadness of the diffraction peaks was ascribed to the small size of the PbSe NCs. ${ }^{14}$ The crystal structure derived from the positions of the wide-range diffraction peaks clearly indicated a cubic rock salt $\mathrm{PbSe}$ phase. From the widths of the diffraction orders, the NCs' sizes are estimated to be around $5 \mathrm{~nm}$, and it is basically consistent with the result of electron microscopy. The inset in Fig. 1 shows a typical TEM micrograph of the corresponding $\mathrm{PbSe} \mathrm{NCs}$ sample, from which the PbSe NCs were assembled into a locally well-ordered close-packed array with a well-controlled size and shape. For example, the NCs' diameter is estimated in Fig. 1 to be $5 \pm 0.5 \mathrm{~nm}$.

Figure 2 shows the absorption spectra and E-A spectra of $\mathrm{PbSe}$ NCs with different sizes in a PMMA film. It is noted that E-A spectra shown in Fig. 2(b) were obtained under the experimental conditions where the angle between the polar-

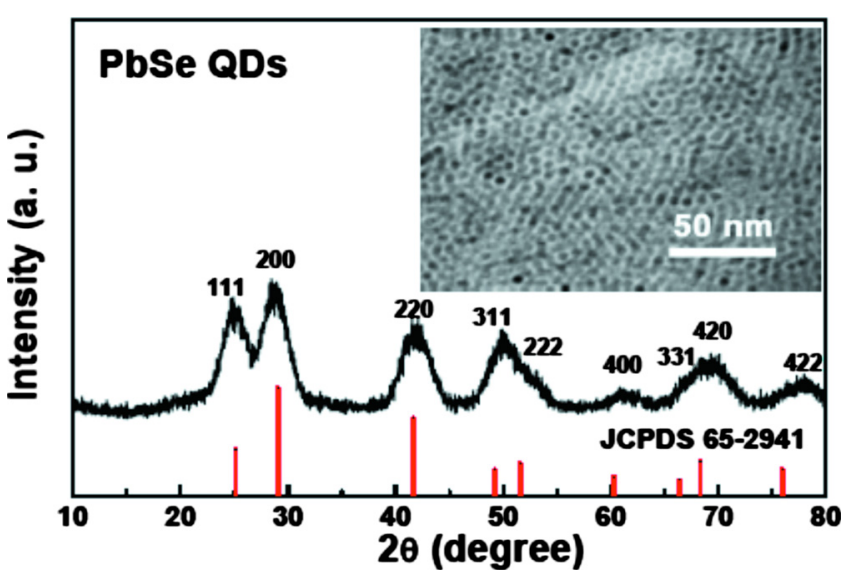

FIG. 1. (Color online) XRD pattern of PbSe QDs and the standard data for PbSe (JCPDS No. 65-2941) as reference. The inset shows TEM micrograph of the corresponding PbSe QDs sample. 

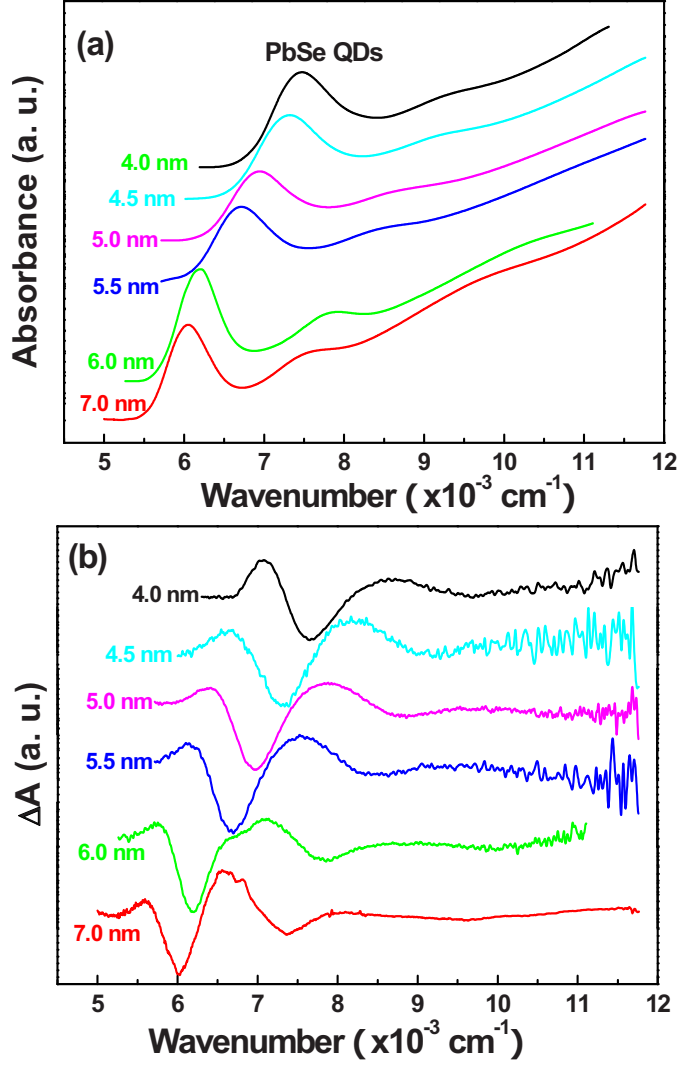

FIG. 2. (Color online) (a) Absorption spectra and (b) E-A spectra for a series of $\mathrm{PbSe}$ QDs with different sizes from 4 to $7 \mathrm{~nm}$ in diameter. The applied electric field was $0.4 \mathrm{MV} \mathrm{cm}^{-1}$ in every case.

ization direction of the excitation light and the direction of the applied electric field, which is hereafter denoted by $\chi$, was $54.7^{\circ}$, i.e., at the magic angle of $\chi$. The E-A spectra shown in Fig. 2 were obtained with application of an ac field having a frequency of $1 \mathrm{kHz}$, and these spectra were independent of the frequency of the applied ac field and the excitation light intensity. As in other QD systems, PbSe NCs exhibit a blueshift in absorption peak with decreasing size, demonstrating a tuning in Fig. 2(a) from $6.05 \times 10^{3} \mathrm{~cm}^{-1}$ $(1653 \mathrm{~nm})$ to $7.63 \times 10^{3} \mathrm{~cm}^{-1}(1310 \mathrm{~nm})$ of a well-resolved lowest-energy exciton transition, as the size decreases from 7 to $4 \mathrm{~nm}$. When electric fields are applied to molecules, each energy level is shifted, depending on the electric dipole moment $(\boldsymbol{\mu})$ and the molecular polarizability $(\boldsymbol{\alpha})$ of the state concerned, i.e., the so-called Stark shift. As the size of the $\mathrm{PbSe} \mathrm{NCs}$ is varied, the Stark signal, i.e., E-A spectrum follows the absorption edge of sample, as shown in Fig. 2(b). The peak of the negative lobes of the E-A spectra is nearly coincident with the peak of the absorption spectrum. Accordingly, the positions of the negative lobes in the Stark spectra show the blueshift, as the size of PbSe NCs decreases.

According to the theory of the electric field effects on optical spectra, ${ }^{15,16}$ the field-induced change in absorption intensity of an isotropically distributed sample at wavenumber $\boldsymbol{\nu}$, i.e., $\Delta A(\boldsymbol{\nu})$, can be given by a sum of the zeroth, first, and second derivations of the absorptions spectrum $A(\boldsymbol{\nu})$ as follows:

$$
\Delta A(\nu)=(f F)^{2}\left[A^{\prime} A(\nu)+B^{\prime} \nu \frac{d\{A(\nu) / \nu\}}{d \nu}+C^{\prime} \nu \frac{d^{2}\{A(\nu) / \nu\}}{d \nu^{2}}\right],
$$

where $f$ is the internal field factor. The coefficient $A^{\prime}$ depends on the field-induced change in transition dipole mo-

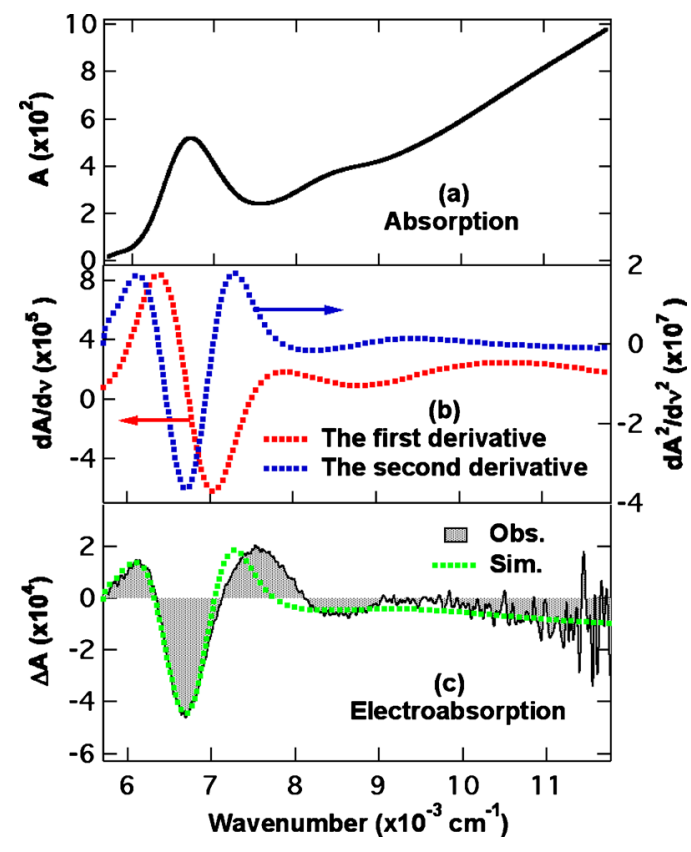

FIG. 3. (Color online) (a) Absorption spectrum of $5.5 \mathrm{~nm}$ diameter $\mathrm{PbSe}$ QDs, (b) the first and second derivative spectra of the absorption spectrum, (c) E-A spectrum (shaded line) observed with a field strength of $0.4 \mathrm{MV} \mathrm{cm}^{-1}$, and the simulated spectrum (dotted line)

ment as well as the field-induced orientation. $B^{\prime}$ is mainly attributed to the difference in molecular polarizability between the ground $(g)$ and excited $(e)$ states, i.e., $\Delta \boldsymbol{\alpha}=\boldsymbol{\alpha}_{\boldsymbol{e}}$ $-\boldsymbol{\alpha}_{g} . C^{\prime}$ depends on the difference in electric dipole moment between the ground and excited states, i.e., $\Delta \boldsymbol{\mu}=\boldsymbol{\mu}_{e}-\boldsymbol{\mu}_{g}$. If the magnitude of $\Delta \boldsymbol{\mu}$ following optical absorption is significant, the presence of $\boldsymbol{F}$ will broaden an isolated transition, giving rise to the E-A spectrum, the shape of which is the second derivative of the absorption spectrum. If the magnitude of $\Delta \boldsymbol{\alpha}$ is significant, the shape of the E-A spectrum is the first derivative of the absorption spectrum. If the transition moment is affected by $\boldsymbol{F}$, the E-A spectrum is similar in shape to the absorption spectrum. The values of $\Delta \boldsymbol{\mu}$ or $\Delta \boldsymbol{\alpha}$ can be obtained from the analysis of the derivative parts of the E-A spectra.

As shown in Fig. 3, E-A spectra of PbSe NCs around the first exciton band are very similar in shape to the second derivative of the absorption spectrum of the corresponding band, suggesting that the field-induced change in absorption spectrum around the first exciton band mainly comes from the change in dipole moment, i.e., $\Delta \boldsymbol{\mu}$, following the transition to the exciton state. The simulation has been done by a linear combination of the zeroth, first, and second derivatives of the absorption spectrum [see Fig. 3(c)].

When the E-A signals at the maxima of the positive and negative in Fig. 3(c) were plotted against the square of the applied field strength, the linear behavior was observed for each peak, indicating that the E-A signal is proportional to the square of the applied field strength. The quadratic field strength dependence is consistent with the one expected from Eq. (1).

The absorption spectra are composed of sharp structured bands superimposed by the broad absorption whose intensity gradually increases with increasing wavenumber [see Figs. 2(a) and 3(a)], and the E-A spectra in the high wavenumber region could not be reproduced well. As far as the first exci- 


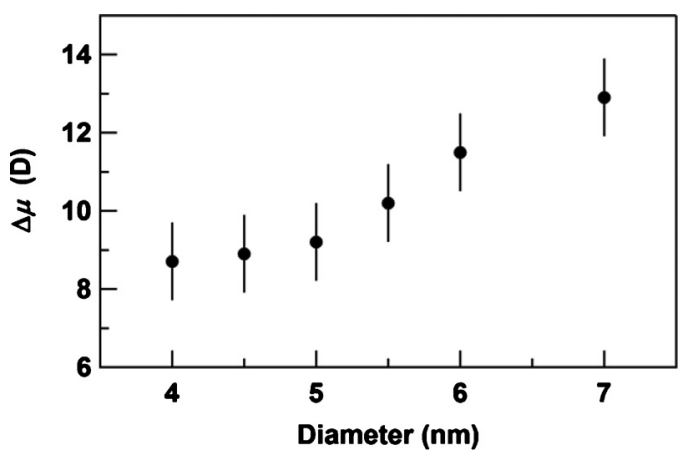

FIG. 4. Plots of the magnitude of $\Delta \boldsymbol{\mu}$ between the first exciton state and the ground state of PbSe QDs in a PMMA film as a function of diameter of nanoparticles. The vertical line shows the error bar.

ton band is concerned, however, the observed E-A spectra could be reproduced quite well by using the derivatives of the observed absorption spectra [see Fig. 3(c) for QDs with a diameter of $5.5 \mathrm{~nm}]$. Then, the magnitude of the change in dipole moment following excitation into the first exciton state has been determined for QDs of PbSe with different sizes. It is noted that $C^{\prime}$ at $\chi=54.7^{\circ}$, i.e., $C_{\chi}^{\prime}$ is given as follows: $C_{\chi}^{\prime}=(\Delta \mu)^{2} / 6 h^{2} c^{2}$, where $h$ and $c$ are Planck's constant and the speed of light, respectively. ${ }^{13}$ As shown in Fig. 4, the magnitude of $\Delta \boldsymbol{\mu}$, thus determined increases, as the particle size increases, indicating that the dipolar character of the first exciton state of $\mathrm{PbSe}$ QDs becomes larger with an increase in the size of QDs. As shown in Fig. 4, the magnitude of $\Delta \boldsymbol{\mu}$ increases nonlinearly with size, suggesting that the electron localization in the exciton state is not proportional to the diameter of QDs. It is also noted that the magnitude of the change in polarizability following absorption to the exciton state is estimated from the first derivative component of the simulated spectrum given in Fig. 3(c) to be $\sim 100 \AA^{3}$ for QDs with a diameter of $5.5 \mathrm{~nm}$.

In conclusion, E-A spectra in the NIR region have been measured for $\mathrm{PbSe} \mathrm{NCs}$ whose size is ranged from 4.0 to
$7.0 \mathrm{~nm}$ in diameter. The E-A spectra primarily come from the change in electric dipole moment following the transition to the exciton state. The magnitude of the change in electric dipole moment following excitation into the first exciton band of PbSe QDs was shown to increase monotonically from 8.7 to $12.9 \mathrm{D}$, as the size of the PbSe QDs increases from 4.0 to $7.0 \mathrm{~nm}$.

X. M. Liu thanks to Japan Society for the Promotion of Science for JSPS Postdoctoral fellowship and for a Grant in Aid for JSPS fellows. This work was supported in part by a Grant-in-Aid for Scientific Research (Grant No. 20043005) from the MEXT in Japan.

${ }^{1}$ J. Robinson, J. Rice, K. Lee, J. Na, R. Taylor, D. Hasko, R. Oliver, M. Kappers, C. Humphreys, and G. Briggs, Appl. Phys. Lett. 86, 213103 (2005)

${ }^{2}$ K. L. Stokes and P. D. Persans, Phys. Rev. B 54, 1892 (1996)

${ }^{3}$ S. A. Empedocles and M. G. Bawendi, Science 278, 2114 (1997).

${ }^{4}$ V. L. Colvin, K. L. Cunningham, and A. P. Alivisatos, J. Chem. Phys. 101, 7122 (1994).

${ }^{5}$ Y. Ohara, T. Nakabayashi, K. Iwasaki, T. Torimoto, B. Ohtani, T. Hiratani, K. Konishi, and N. Ohta, J. Phys. Chem. B 110, 20927 (2006).

${ }^{6}$ E. Menéndez-Proupin and C. Trallero-Giner, Phys. Rev. B 69, 125336 (2004).

${ }^{7}$ E. H. Sargent, Adv. Mater. (Weinheim, Ger.) 17, 515 (2005).

${ }^{8}$ M. A. Hines and G. D. Scholes, Adv. Mater. (Weinheim, Ger.) 15, 1844 (2003).

${ }^{9}$ T. Mokari, M. Zhang, and P. Yang, J. Am. Chem. Soc. 129, 9864 (2007).

${ }^{10}$ H. Du, C. Chen, R. Krishnan, T. D. Krauss, J. M. Harbold, F. W. Wise, M. G. Thomas, and J. Silcox, Nano Lett. 2, 1321 (2002).

${ }^{11}$ R. Ellingson, M. Beard, J. C. Johnson, P. Yu, O. Micic, A. Nozik, A. Shabaev, and A. Efros, Nano Lett. 5, 865 (2005).

${ }^{12}$ W. W. Yu, J. C. Falkner, B. S. Shih, and V. L. Colvin, Chem. Mater. 16, 3318 (2004)

${ }^{13}$ J. Tayama, T. Iimori, and N. Ohta, J. Chem. Phys. 131, 244509 (2009).

${ }^{14}$ A. L. Rogach, A. Kornowski, M. Gao, A. Eychmueller, and H. Weller, J. Phys. Chem. B 103, 3065 (1999).

${ }^{15}$ W. Liptay, in Excited States, edited by E. C. Lim (Academic, New York, 1974), Vol. 1, p. 129.

${ }^{16}$ E. Jalviste and N. Ohta, J. Photochem. Photobiol. C 8, 30 (2007). 